\title{
Synthesis and biological characterisation of ester and amide derivatives of Fusidic acid as antiplasmodial agents
}

Kawaljit Singh $^{\mathrm{a},}$, Marlene Espinoza-Moraga ${ }^{\mathrm{a},}$, Mathew Njoroge ${ }^{\mathrm{a},}$, Gurminder Kaur ${ }^{\mathrm{a},}$, John

Okombo $^{\mathrm{a},}$,Carmen De Kock ${ }^{\mathrm{c}}$, Peter J. Smith ${ }^{\mathrm{c}}$, Sergio Wittlin ${ }^{\mathrm{d}, \mathrm{e}}$ and Kelly Chibale ${ }^{\mathrm{a}, \mathrm{b}, \mathrm{f},{ }^{*}}$

aDepartment of Chemistry, University of Cape Town, Rondebosch 7701, South Africa

${ }^{\mathrm{b}}$ South African Medical Research Council Drug Discovery and Development Research Unit, University of Cape Town, Rondebosch 7701, South Africa

${ }^{c}$ Division of Clinical Pharmacology, Faculty of Health Sciences, University of Cape Town, K45, OMB, Groote Schuur Hospital, Observatory, 7925, South Africa

${ }^{\mathrm{d}}$ Swiss Tropical and Public Health Institute, Socinstrasse 57, 4002 Basel, Switzerland

eUniversity of Basel, 4002 Basel, Switzerland

fInstitute of Infectious Disease and Molecular Medicine, University of Cape Town, Rondebosch 7701, South Africa

Abstract: A series of novel fusidic acid (FA) derivatives was synthesized by replacing the carboxylic acid group with various ester and amide groups and evaluated in vitro for their antiplasmodial activity against the chloroquine-sensitive NF54 and multidrugresistant K1 strains of the malarial parasite Plasmodium falciparum. Most of these derivatives showed a 4-49 and 5-17 fold increase in activity against NF54 and KI strains, respectively, as compared to FA and had a good selectivity index. These derivatives are stable over the incubation period and do not appear to be prodrugs of fusidic acid.

Malaria persists as a major public health problem, resulting in 214 million cases and causing 438,000 deaths worldwide in 2015. ${ }^{1}$ The disease, transmitted by female Anopheles mosquitoes, is caused by five different species of the protozoan Plasmodium parasite, namely: P.falciparum, P.vivax, P.malariae, P.ovale, and P.knowlesi that infect and destroy red blood cells leading to high fever, anaemia, cerebral malaria, and possibly death. Of these, $P$. falciparum is the most lethal as well as the most prevalent in sub-Saharan Africa and is responsible for high mortality rates especially amongst young children and pregnant women. As there is no available vaccine, the control of the disease relies largely on the use of bed nets, other individual protection against mosquito bites, and the successful drug treatment of infected patients. ${ }^{2}$ The rapid development of drug resistance has compromised the use of previously 
effective drugs such as chloroquine, sulfadoxine/pyrimethamine and artemisinin. ${ }^{3,4}$ In this context, the discovery and development of new molecules with novel mechanisms of action and no cross-resistance to current drugs is necessary.

Nature has always been a rich source of potential antimalarial agents. Two of the most effective antimalarial drugs quinine and artemisinin are natural products isolated from Cinchona officinalis and Artemisia annua, respectively, and many of the synthetic antimalarial drugs are analogues of these two natural products. ${ }^{5-8}$ Fusidic acid (FA) (Figure 1) is a steroid-based, narrow spectrum bacteriostatic antibiotic belonging to a family of naturally occurring antibiotics, the fusidanes, which have a tetracyclic ring system with a unique chair-boat-chair conformation. It was first isolated from the fungus Fusidium coccineum in 1960 and has been in clinical use since 1962 for the treatment of skin, bone and joint infections caused by Staphylococcus aureus as well as against several other Gram-positive species. ${ }^{9-11}$ The in vitro $\mathrm{MIC}_{50}$ of FA against most gram-positive bacteria lies between $0.1 \mu \mathrm{M}-1 \mu \mathrm{M} .{ }^{12-14}$ Its proposed mechanism of action (MoA) involves inhibition of bacterial protein synthesis by locking elongation factor-G (EF-G) on the ribosome with Guanosine 5'-diphosphate (GDP) in a posttranslocational state. ${ }^{15,16}$ The clinical value of FA is attributed to its excellent tissue distribution, low degree of toxicity and allergic reactions, excellent oral bioavailability, and the absence of cross-resistance with other clinically used antibiotics. ${ }^{17,18}$

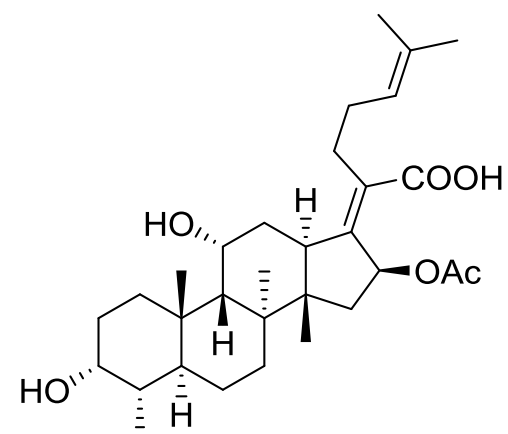

Figure 1. Structure of fusidic acid

FA has also been reported to have in vitro antiplasmodial activity, with a reported $\mathrm{IC}_{50}$ of about $50 \mu \mathrm{M}$ against chloroquine-sensitive $P$. falciparum line D10. ${ }^{19,20}$ Its MoA has been proposed to be through inhibition of plasmodial EF-Gs, which harbour bacteria-like metabolic processes and are localised within the parasite's mitochondria and the relict apicoplast. ${ }^{20,21}$

We have been exploring the antiplasmodial potential of FA and its derivatives. Recently, we identified a number of FA derivatives with better (2-35 fold relative to FA) antiplasmodial activity by replacing its carboxylic acid with various bioisosteres. ${ }^{22}$ In the 
present investigation, we report the synthesis and biological evaluation of novel aliphatic as well as aromatic ester and amide analoguesof FA.

The compounds synthesized for this study are presented in Table 1 with the corresponding synthetic routes outlined in Scheme 1. Target compounds were synthesized following a straightforward synthetic route involving a single step from commercially available fusidic acid 1 (Scheme 1). Aryl esters of fusidic acid 2a-o were obtained in moderate to good yield (38-89\%, Table 1) by reacting 1 with corresponding alcohols (ROH) using N,N'dicyclohexylcarbodiimide (DCC) as a coupling agent in the presence of a catalytic amount of 4-dimethylaminopyridine (DMAP) in dichloromethane. Aliphatic ester 2p-u were obtained through 1-ethyl-3-(3-dimethylaminopropyl)carbodiimide (EDCI)-mediated coupling of 1 with corresponding alcohols in relatively poor yield (9-37\%, Table 1). EDCI mediated coupling of $\mathbf{1}$ with corresponding amines on the other hand, afforded aromatic (2v) and aliphatic (2w-y) amide derivatives in moderate to good yields (31-73\%, Table 1). All the target compounds were purified using column chromatography and fully characterized by a range of analytical and spectroscopic techniques (see ESI $\dagger$ ).
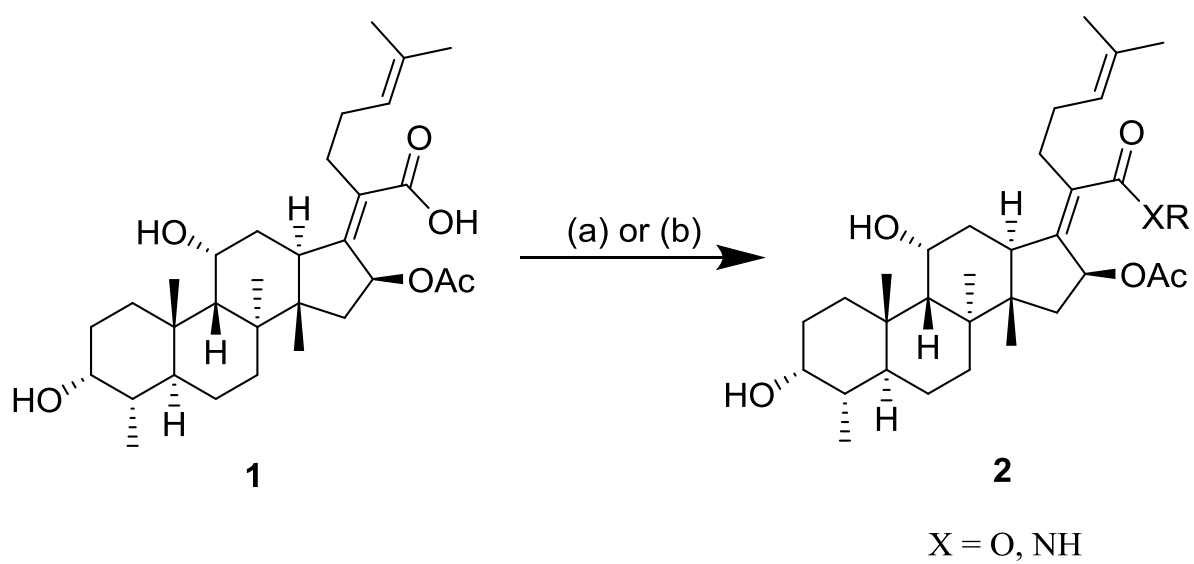

Scheme 1. Reagents andreaction conditions: (a) ROH (1.3 equiv.), DCC (1.3 equiv.), DMAP, DCM (15 ml), 2 h, $25^{\circ} \mathrm{C}$; (b) ROH/RNH 2 (1.2 equiv.), EDCI (2.5 equiv.), DMAP (2.5 equiv.), $\operatorname{DCM}(6 \mathrm{ml}), 12 \mathrm{~h}, 25^{\circ} \mathrm{C}$.

The synthesized compounds were evaluated for their in vitro antiplasmodial activity against the chloroquine-sensitive NF54 strain, using the lactate dehydrogenase assay, and the multidrug-resistant K1 strain, using the modified $\left[{ }^{3} \mathrm{H}\right]$ hypoxanthine incorporation assay. ${ }^{23,24}$ Chloroquine and artesunate were used as the reference drugs in all the experiments. 
Compounds were also evaluated for cytotoxicity against a mammalian cell-line, Chinese Hamster Ovarian (CHO) cells.

The in vitro antiplasmodial activity and cytotoxicity data as indicated by their $\mathrm{IC}_{50}$ values is summarized in Table 1. Most of these analogues were equipotent against both strains (NF54 and K1). The IC50 of FA against NF54 and K1strains was 59.0 and $19.0 \mu \mathrm{M}$, respectively. 21 out of 25 compounds (2a-r, $2 \mathbf{v}-\mathbf{x}$, Table 1) displayed superior potency compared to FA against both NF54 (4-49 fold) and K1(5-17 fold) strains with $\mathrm{IC}_{50} \leq 13.4 \mu \mathrm{M}$ and $\leq 4.0 \mu \mathrm{M}$, respectively.

The potencies of $\mathbf{2 a - 0}$ indicated that aromatic ester derivatives were well tolerated. Unsubstituted aromatic ester (2a, Table 1) as well as ester derivatives substituted with electronwithdrawing groups (EWG), fluoro (2b), chloro (2c) and bromo (2d) in the para-position resulted in 20-28-fold and 12-14-fold enhancement in antiplasmodial activity relative to FA against both P.falciparum NF54 and K1. Varying the position of the substituent from para- to meta-on the phenyl ring did not have a significant effect on antiplasmodial activity against both the strains ( $\mathbf{2 b}, \mathbf{2 c}$ and $\mathbf{2 d}$ vs $\mathbf{2 e}, \mathbf{2} \mathbf{f}$ and $\mathbf{2 g}$, respectively) as all of these derivatives retained comparable activity. Likewise, introduction of an electron-donating group (EDG), -OMe in the meta-position (2h) resulted in similar activity values (Table 1). Further, disubstituted derivatives (2i-n) retained good antiplasmodial activity. Among dichloro-substituted derivatives (2i-k), 3,5-disubstituted ester analogue (2j) was found to have superior activity against both strains (NF54, IC $50=2.0 \mu \mathrm{M} ; \mathrm{K} 1, \mathrm{IC}_{50}=1.1 \mu \mathrm{M}$ ) in comparison to its 3,4disubstituted (2i) and 2,4-disubstituted (2k) analogues. The activity of 4-chloro-substituted derivative (2c) was also compared with its regioisomer, 3-chloro (2f) as well as the 3,4-dichloro (2i), 3,5-dichloro (2j) and 2,4-dichlorosubstituted (2k) ester derivatives; compound $\mathbf{2} \mathbf{f}$ was found to be the most active amongst them with an $\mathrm{IC}_{50}$ value of $1.4 \mu \mathrm{M}$ and $1.2 \mu \mathrm{M}$ against NF54 and K1 strain, respectively. The comparison of 3,5-dichlorosubstituted (2j) analogue with 3,5-difluorosubstituted (2I) ones revealed higher activity for the former. Ester analogues of fusidic acid having the phenyl ring disubstituted with electron releasing -Me group at the 2,5- (2m, NF54, IC $503.0 \mu \mathrm{M}$; KI, IC $501.4 \mu \mathrm{M})$ and 2,4-positions (2n, NF54, IC $502.4 \mu \mathrm{M}$; KI, $\left.\mathrm{IC}_{50} 1.4 \mu \mathrm{M}\right)$ also retained potency against both strains. It can be concluded from these results that the electronic properties of the substituents do not have a significant impact on the antiplasmodial activity of these compounds. Attempts at replacing the phenyl ring with a naphthyl group yielded ester analogue (2o) having similar activity. The effect of aliphatic esters with varying carbon chain lengths on antiplasmodial activity was also established wherein it was observed that an increase in carbon chain length decreased the activity. Although 
derivatives 2p-r demonstrated superior antiplasmodial activity (NF54, IC 50 3.4-4.6 $\mu \mathrm{M}$; KI, $\left.\mathrm{IC}_{50} 1.5-2.2 \mu \mathrm{M}\right)$ as compared to fusidic acid, lengthening of the alkyl chain (2s-t, Table 1), diminished their corresponding activities. All these aryl and alkyl ester derivatives (2a-u) were also tested for cytotoxicity and were found to be non-cytotoxic, as indicated by their high $\mathrm{IC}_{50}$ values against $\mathrm{CHO}$ cell lines as well as their high selectivity index values (Table 1). Replacing an ester with an amide functionality seemed to have no effect on activity as aromatic amide derivative also retained the antiplasmodial activity (2v vs $\mathbf{2 a}$, Table 1$)$. However, long chain alkyl amide derivatives were found to be active, in striking contrast to their ester counterparts (2w and $\mathbf{2 x}$ vs $\mathbf{2 s}$ and $\mathbf{2 t}$, respectively, Table 1). Amide derivatives also displayed favourable toxicity profiles as evident from their high SI values.

Table 1. Yields, in vitro antiplasmodial activity of compounds against NF54 and K1 strain of $P$. falciparum, cytotoxicity against $\mathrm{CHO}$ cell lines and selectivity index.

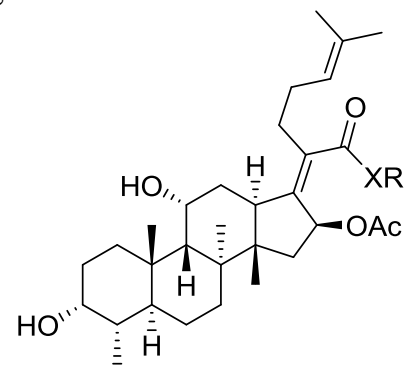

\begin{tabular}{|c|c|c|c|c|c|c|c|}
\hline \multirow{2}{*}{ Compound } & \multirow{2}{*}{$\mathbf{X}$} & \multirow{2}{*}{$\mathbf{R}$} & \multirow{2}{*}{$\begin{array}{l}\text { Yield } \\
(\%)\end{array}$} & \multicolumn{2}{|c|}{$\begin{array}{l}\text { Antiplasmodial } \\
\text { activity }\end{array}$} & \multirow{2}{*}{$\begin{array}{c}\text { Cytotoxicity } \\
\text { (CHO) IC I0, } \\
\boldsymbol{\mu M}\end{array}$} & \multirow[b]{2}{*}{$\mathbf{S I}^{a}$} \\
\hline & & & & $\begin{array}{c}\text { NF54 } \\
\text { IC } 50, \mu M\end{array}$ & $\begin{array}{c}\text { K1 } \\
\text { IC } 50, \mu M\end{array}$ & & \\
\hline Fusidic acid & & & & 59.0 & 19.0 & $>194$ & $>3$ \\
\hline $2 a$ & $\mathrm{O}$ & & 55 & 2.4 & 1.4 & 99.4 & 41 \\
\hline $2 b$ & $\mathrm{O}$ & & 38 & 2.9 & 1.4 & 79.4 & 27 \\
\hline $2 c$ & $\mathrm{O}$ & & 89 & 2.3 & 1.4 & 71.6 & 31 \\
\hline $2 d$ & $\mathrm{O}$ & & 52 & 2.1 & 1.6 & 95.3 & 45 \\
\hline $2 e$ & $\mathrm{O}$ & & 88 & 2.6 & 2.0 & 89.4 & 34 \\
\hline $2 f$ & $\mathrm{O}$ & & 61 & 1.4 & 1.2 & 87.7 & 63 \\
\hline $2 g$ & $\mathrm{O}$ & & 51 & 2.6 & 1.6 & 98.3 & 38 \\
\hline $2 \mathrm{~h}$ & $\mathrm{O}$ & & 88 & 1.8 & 1.3 & 86.4 & 48 \\
\hline $2 \mathbf{i}$ & $\mathrm{O}$ & & 54 & 3.6 & 2.2 & 95.0 & 26 \\
\hline
\end{tabular}




\begin{tabular}{lllllll}
\hline $\mathbf{2 j}$ & $\mathrm{O}$ & & & & \\
& & & & & \\
\end{tabular}

$\mathrm{ND}=$ not determined. ${ }^{a} \mathrm{SI}($ selectivity index $)=\mathrm{IC}_{50} \mathrm{CHO} / \mathrm{IC}_{50} \mathrm{NF} 54$.

Plasmodial parasites are known to possess esterase enzymes which can hydrolyse prodrugs to their active forms. ${ }^{25} \mathrm{We}$ therefore investigated whether these compounds were being hydrolysed to FA during incubation. The experiment was designed with the same setup as the $\mathrm{IC}_{50}$ determination, but with sampling at various time points during the incubation (see ESI $\dagger$ ). FA was not detected in any of the incubations and the relative compound concentration remained constant throughout the 48-hour incubation. This suggests that these derivatives are not prodrugs of FA but are actually active in their original form. This finding is in line with our previous work on FA bioisosteres where we observed better antiplasmodial activity with the more lipophilic carboxylic acid bioisosteres. It also confirms our previous finding that a free carboxylic acid group is not essential for antiplasmodial activity. This is distinct from the antibacterial activity of FA analogues where activity is lost on substitution of the acid, and suggests differences in the targets and/or MoA between bacteria and plasmodia. ${ }^{26,27}$ 
In conclusion, a series of novel FA derivatives have been synthesized by replacing the carboxylic acid group with aliphatic as well as aromatic ester and amide groups, and evaluated in vitro for their antiplasmodial activity against NF54 and K1 strains of P. falciparum. Almost all of these analogues exhibited improved antiplasmodial activity relative to FA against both strains with good selectivity index values. The enhanced antiplasmodial activity substantiates the insignificance of a free carboxylic group for antiplasmodial activity. In addition, these derivatives were stable over the incubation period and were not converted to fusidic acid under the incubation conditions used and are therefore unlikely to act as prodrugs. Overall this work demonstrates the potential of FA as a promising novel antimalarial template.

\section{Acknowledgements}

The University of Cape Town, South African Medical Research Council, and South African Research Chairs initiative of the Department of Science and Technology administered through the South African National Research Foundation are gratefully acknowledged for support $(\mathrm{KC})$.

\section{References}

1. World Health Organisation World Malaria Report 2015; Geneva, 2015.

2. World Health Organization Guidelines for the treatment of malaria; 3rd ed.; Geneva, 2015. 
3. White, N. J. J. Clin. Invest. 2004, 113, 1084-1092.

4. Dondorp, A. M.; Nosten, F.; Yi, P.; Das, D.; Phyo, A. P.; Tarning, J.; Lwin, K. M.;

Ariey, F.; Hanpithakpong, W.; Lee, S. J.; Ringwald, P.; Silamut, K.; Imwong, M.;

Chotivanich, K.; Lim, P.; Herdman, T.; An, S. S.; Yeung, S.; Singhasivanon, P.; Day, N. P. J.; Lindegardh, N.; Socheat, D.; White, N. J. N. Engl. J. Med. 2009, 361, 455-67.

5. Negi, A. S.; Gupta, A.; Hamid, A. A. Curr. Med. Chem. 2014, 21, 458-500.

6. Guantai, E.; Chibale, K. Malar. J. 2011, 10 Suppl 1, S2.

7. Wells, T. N. Malar. J. 2011, 10 Suppl 1, S3.

8. $\quad$ Nogueira, C. R.; Lopes, L. M. X. Molecules 2011, 16, 2146-2190.

9. Godtfredsen, W. O.; Janhsen, S.; Lorck, H.; Roholt, K.; Tybring, L. Nature 1962, 193, 987.

10. Godtfredsen, W.; Roholt, K.; Tybring, L. Lancet 1962, 279, 928-931.

11. Collignon, P.; Turnidge, J. Int. J. Antimicrob. Agents 1999, 12, S45-S58.

12. Toma, E.; Barriault, D. J. Clin. Microbiol. 1995, 33, 1712-5.

13. Pfaller, M. A.; Castanheira, M.; Sader, H. S.; Jones, R. N. Int. J. Antimicrob. Agents 2010, 35, 282-7.

14. Jones, R. N.; Mendes, R. E.; Sader, H. S.; Castanheira, M. Clin. Infect. Dis. 2011, 52 Suppl 7, S477-86.

15. Harvey, C. L.; Knight, S. G.; Sih, C. J. Biochemistry 1966, 5, 3320-3327.

16. Kinoshita, T.; Kawano, G.; Tanaka, N. Biochem. Biophys. Res. Commun. 1968, 33, 769-773.

17. Turnidge, J. Int. J. Antimicrob. Agents 1999, 12 Suppl 2, S23-34.

18. Kraus, C. N.; Burnstead, B. W. Clin. Infect. Dis. 2011, 52 Suppl 7, S527-37.

19. Black, F.; Wildfang, I.; Borgbjerg, K. Lancet 1985, 325, 578-579.

20. Johnson, R. A.; McFadden, G. I.; Goodman, C. D. PLoS One 2011, 6, e20633. 
21. Gupta, A.; Mir, S. S.; Saqib, U.; Biswas, S.; Vaishya, S.; Srivastava, K.; Siddiqi, M. I.; Habib, S. Mol. Biochem. Parasitol. 2013, 192, 39-48.

22. Kaur, G.; Singh, K.; Pavadai, E.; Njoroge, M.; Espinoza-Moraga, M.; De Kock, C.; Smith, P. J.; Wittlin, S.; Chibale, K. Med. Chem. Commun. 2015, 6, 2023-2028.

23. Makler, M. T.; Ries, J. M.; Williams, J. A.; Bancroft, J. E.; Piper, R. C.; Gibbins, B. L.; Hinrichs, D. J. Am. J. Trop. Med. Hyg. 1993, 48, 739-41.

24. Vennerstrom, J. L.; Arbe-Barnes, S.; Brun, R.; Charman, S. A.; Chiu, F. C. K.;

Chollet, J.; Dong, Y.; Dorn, A.; Hunziker, D.; Matile, H.; McIntosh, K.;

Padmanilayam, M.; Santo Tomas, J.; Scheurer, C.; Scorneaux, B.; Tang, Y.; Urwyler, H.; Wittlin, S.; Charman, W. N. Nature 2004, 430, 900-904.

25. Mishra, S.; Karmodiya, K.; Parasuraman, P.; Surolia, A.; Surolia, N. Bioorg. Med. Chem. 2008, 16, 5536-46.

26. Godtfredsen, W. O.; Von Daehne, W.; Tybring, L.; Vangedal, S. J. Med. Chem. 1966, $9,15-22$.

27. Von Daehne, W.; Godtfredsen, W. O.; Rasmussen, P. R. In Advances in applied microbiology; 1979; Vol. 25, pp. 95-146. 